\title{
Estudio comparativo de normas para el análisis dinámico de una torre autosoportada bajo carga de viento
}

\section{Comparative study of standards for the dynamic analysis of a self-supporting tower under wind load}

\author{
P. Martín ${ }^{(*)}$, I. Fernández ${ }^{(*)}$, V. E. Parnás ${ }^{(*)}$
}

\section{RESUMEN}

El estudio de los métodos de análisis dinámicos para la consideración de la carga de viento es de gran importancia en las torres autosoportadas de telecomunicaciones debido a que son estructuras altamente vulnerables a los vientos extremos. En Cuba el análisis de las torres autosoportadas se realiza según lo establecido en la norma cubana de viento NC-285:2003 (NC). Esta norma es de carácter general para todas las edificaciones y no presenta las particularidades del análisis de las torres reticuladas bajo carga de viento por esta razón es necesario evaluar sus diferencias con respecto a otras normas internacionales. El presente trabajo tiene como objetivo comparar los valores de fuerzas en los elementos y desplazamientos en una torre autosoportada obtenidos de la aplicación de los métodos de análisis propuestos en las normas NC, ISO 43542009 (ISO), AS/NZS1170.2-2011 (AS/NZ) y EC 3: 2007 (EC3). La comparación entre las normas arrojó como resultado que las fuerzas axiales y desplazamientos obtenidos por la norma $\mathrm{NC}$ son menores que las obtenidos por las normas ISO, AS/NZ, EC3.

Palabras clave: torres autosoportadas, carga de viento, normas, análisis dinámico.

\section{ABSTRACT}

The study of methods of analysis for consideration of the wind load is very important in telecommunications towers because they are highly vulnerable structures due to extreme wind actions. In Cuba the analysis of self-supporting towers is carried out as is defined on the wind Cuban code NC-285: 2003 (NC). This code does not present the specifications of the effects of wind on self-supporting towers, for that reason there is a necessity to evaluate its differences with others international codes and standards. The aim of this paper is to compare the element forces and displacements of a selfsupporting towers which were obtained from the application of the analysis methods enclosed in the codes and standards NC, ISO 4354-2009 (ISO), AS/NZS1170.2-2011 (AS/NZ) and EC 3: 2007 (EC3). The results showed that axial forces on elements and displacements of the tower obtained from application of NC were lower than those obtained from application of ISO, AS/NZ, EC3.

Keywords: Self-supporting towers, wind load, codes and standards, dynamic analysis.

(*) Universidad Tecnológica de La Habana “José Antonio Echeverría”, La Habana, (Cuba) Persona de contacto/Corresponding author: vivian@civil.cujae.edu.cu (V. Elena Parnás) ORCID: http://orcid.org/oooo-0002-8954-5159 (P. Martín); http://orcid.org/oooo-0oo2-1223-0968 (I. Fernández); http://orcid.org/oooo-0oo1-7912-7570 (V. E. Parnás)

Cómo citar este artículo/Citation: P. Martín, I. Fernández, V. E. Parnás (2018). Estudio comparativo de normas para el análisis dinámico de una torre autosoportada bajo carga de viento. Informes de la Construcción, 70(552): e274. https://doi.org/10.3989/ic.15.021 Copyright: (c) 2018 CSIC. Este es un artículo de acceso abierto distribuido bajo los términos de la licencia de uso y distribución Creative Commons Reconocimiento 4.0 Internacional (CC BY 4.0). 


\section{INTRODUCCIÓN}

Las torres de telecomunicaciones son estructuras altamente vulnerables a la acción de la carga de viento. El fallo de las torres de telecomunicaciones origina graves pérdidas económicas y sociales que se manifiestan, entre otros aspectos, por la falta de comunicación en momentos de desastres. Por esta razón, el estudio de la acción del viento sobre las torres de telecomunicaciones tiene una gran importancia para cuba donde frecuentemente se producen vientos extremos debido al paso de huracanes. Solo en el período del 1996 al 2012 fallaron en cuba 59 torres de telecomunicaciones. Uno de los aspectos a revisar para evitar los fallos son los métodos de cálculo usados para el análisis de las torres de telecomunicaciones, ya que estos han evolucionado en el tiempo (1), (2), (3). El estado actual del conocimiento, los costos y la infraestructura necesaria para el desarrollo de investigaciones experimentales en el estudio de las torres autosoportadas de telecomunicaciones bajo carga de viento, hace conveniente prestar atención a los métodos analíticos para la consideración de la carga de viento en esta tipología estructural.

La interacción del viento sobre la estructura puede ser modelada en tres componentes de la respuesta estructural: longitudinal, transversal y torsional. El presente artículo se orienta al estudio de la obtención de la respuesta longitudinal de las torres autosoportadas bajo carga de viento. Las fuerzas interiores que actúan en las torres autosoportadas son fundamentalmente de carácter semiestáticos correspondientes a la componente media de la velocidad del viento y efectos dinámicos correspondientes a la componente fluctuante de la velocidad del viento. Investigaciones realizadas en este tema (2), (4), (5) establecen que los efectos dinámicos pueden ser predominantemente no resonantes cuando la mayor parte de la energía contenida en el espectro de respuesta está por debajo de la menor frecuencia de vibración natural de la estructura y predominantemente resonantes cuando la energía contiene las frecuencias naturales de vibración de la estructura.

Los métodos para el análisis de las estructuras esbeltas bajo carga de viento, incluyendo las torres autosoportadas de celosía, se basan en definir los procedimientos para tomar en cuenta cada una de las componentes de la respuesta de la estructura (media, no resonante y resonante) (1), (2), (3). Todos los métodos incluyen el análisis de la respuesta media de la estructura, es decir, la componente estática, sin embargo la consideración de la componente dinámica, que incluye la respuesta resonante y no resonante, varía en función de las características de la estructura. La respuesta resonante de las estructuras es importante cuando las frecuencias naturales de vibración son menores de $1 \mathrm{hz}$ porque en esa faja de frecuencias la energía de las ráfagas del viento es mayor. Los métodos identificados en la bibliografía para el análisis de las torres autosoportadas son el método estático conocido como método "factor de velocidad de ráfaga" y los métodos dinámicos "factor de respuesta de ráfaga" y el método de "líneas de influencia". El método de "líneas de influencia" no se encuentra incluido en las normas internacionales de cálculo debido a que es muy engorrosa su aplicación. El método más aplicado internacionalmente para el análisis de estructuras sensibles a las acciones dinámicas de la carga de viento es el método "factor de respuesta de ráfaga" desarrollado originalmente por davenport (1) a inicios de la década del sesenta. El método utiliza la teoría de vibraciones estocásticas para estimar las cargas inducidas por el viento y la respuesta dinámica longitudinal de la estructura. Davenport (1) expuso que los desplazamientos máximos de la estructura debido al viento podían ser determinados multiplicando los desplazamientos estáticos medios por el factor de respuesta de ráfaga. Las hipótesis de davenport para la definición del método fueron: a) comportamiento estático lineal de la estructura; b) modelación de la estructura como un oscilador de un grado de libertad; c) turbulencia idealizada como un proceso gaussiano estacionario con media igual a cero; d) el cuadrado de la turbulencia es despreciado en la expresión de la presión del viento. Desde la década del setenta y hasta los años 2000 se realizaron varios trabajos (3), (6), (7) donde se perfecciona el método de "factor de respuesta de ráfaga" propuesto por davenport (1), y se proporcionan fórmulas simplificadas para su aplicación que fueron incluidas posteriormente en las principales normas de acción del viento sobre las estructuras en el mundo (8), (9), (10), (11), (12), (13). Algunas de estas normas proponen el método de forma idéntica a la expuesta por davenport y otras normas realizan algunas modificaciones, pero los fundamentos teóricos son los mismos. Una de las variaciones del método está en la multiplicación del factor de ráfaga por la fuerza media del viento y no por la respuesta media de la estructura, por esta razón el método ha adoptado el nombre "factor de efecto de ráfaga" (gef-siglas en inglés), ya que puede estar modificando tanto la respuesta como la fuerza del viento. Otra de las modificaciones realizadas al método "factor de respuesta de ráfaga" fue realizada por zhou y kareem (3), quienes tuvieron en cuenta la forma no lineal del primer modo de oscilación.

Las particularidades de la obtención del gef en torres autosoportadas de celosía fueron estudiadas por holmes (14), (15) en los años noventa, quien tuvo en cuenta la curvatura en la forma del primer modo de oscilaciones y la forma troncopiramidal de las torres. Holmes obtuvo las expresiones para el cálculo del amortiguamiento aerodinámico y estudió la influencia de la variación de la velocidad media en el cálculo del factor de respuesta de ráfaga, donde llegó a la conclusión que la respuesta resonante se incrementa significativamente a medida que aumenta la velocidad media del viento. Otra particularidad en el análisis es la consideración de los efectos dinámicos en los elementos diagonales de las torres que presentan configuración tipo eiffel (16) a partir de la definición de patrones de carga media y fluctuante sobre la torre. Los resultados de los estudios anteriores se encuentran incluidos en las normas as3995-1994 (17) y ec 3: 2007 (ec3) (18). Las normas canadiense (19) y estadounidense (20) de diseño de torres de telecomunicaciones presentan la aplicación de un factor de ráfaga para el análisis de las torres autosoportadas, pero no expone los detalles de la metodología que debe aplicarse para la obtención de este factor.

Cuba no presenta una norma específica para el análisis y diseño de las torres de telecomunicaciones bajo carga de viento, por tanto debe aplicarse lo establecido en la norma cubana de viento para edificaciones nc-285:2003 (nc) (21). Esta norma propone un método para la consideración de la componente dinámica de la carga de viento basado en estudios realizados en la antigua unión soviética, el método es similar al expuesto en la norma rusa de cargas en estructuras (22). La norma nc es de obligatorio cumplimiento en el país, sin embargo es posible la aplicación del método propuesto en la norma internacional iso 4354-2009 (iso) (12) por ser una norma de carácter internacional. Las normas nc e iso 
no presentan las particularidades expresadas en los trabajos mencionados anteriormente para la obtención de la respuesta longitudinal de las torres autosoportadas frente a carga de viento, por tal razón es necesario evaluar sus diferencias con respecto a los métodos propuestos en las normas que consideran las especificaciones de las torres de telecomunicaciones.

El presente trabajo tiene como objetivo comparar los valores de fuerzas en los elementos y desplazamientos en una torre autosoportada obtenidos de la aplicación de los métodos de análisis propuestos en las normas nc (21), iso (12), as/nz (9) y ec3 (18). La norma australiana de viento as/nzs1170.2-2011 (as/nz) (9) fue seleccionada para el presente estudio ya que presenta el procedimiento para el análisis de la respuesta longitudinal de las torres autosoportadas bajo carga de viento y actualiza los parámetros que describen la respuesta no resonante de la torre con respecto a la norma australiana específica de torres as3995-1994 (17). En el trabajo se describen inicialmente las características estructurales y geométricas de la torre autosoportada objeto de estudio, así como los bases definidas para su modelación computacional (sección 2.1). Los parámetros meteorológicos de la carga de viento, tales como perfil de velocidades medias, espectro, escala integral e intensidad de turbulencia son expuestos en la sección 2.2. La sección 2.3 describe los métodos para la obtención de la respuesta longitudinal de la torre autosoportada según las normas seleccionadas para este estudio. Las comparaciones de los parámetros de los métodos definidos en las normas, así como las comparaciones de las fuerzas axiales en los elementos y desplazamientos de la torre son mostradas en la sección 3.

\section{METODOLOGÍA}

\subsection{Modelación de la estructura y análisis modal}

La torre autosoportada de telecomunicaciones seleccionada para el estudio es de diseño y fabricación cubana. Esta torre es de sección transversal cuadrada, el ancho de la base es de 6 metros y presenta forma troncopiramidal hasta los 45 metros de altura, donde comienza una torreta de sección transversal constante de 1,17 metros hasta los 60 metros. Sobre la torreta se encuentra un mástil de sección circular de 6 metros. Los elementos principales que conforman la torre son columnas (c), diagonales (d) y tranques (t), ver figura 1. La torre presenta 24 paneles uhf colocados en el mástil de la torre y 12 paneles vhf colocados en la torreta.

La modelación y análisis de la torre se realizó utilizando como herramienta el programa SAP 2000 (versión 14) basado en el método de elementos finitos. Fue realizado un análisis lineal geométrico ya que el comportamiento de las torres autosoportadas es lineal (5), (16). La torre fue modelada como una armadura espacial, cada miembro de la estructura tridimensional fue reproducido de forma detallada. Los elementos de la armadura fueron modelados como barras, elementos lineales con dos nudos extremos. Las columnas se consideraron continuas desde la base hasta la cima, ellas se encuentran arriostradas por los tranques principales los cuales disminuyen la longitud de pandeo del elemento y rigidizan la estructura. Las uniones entre los elementos diagonales y tranques con las columnas fueron consideradas articuladas. El material que conforma los elementos de las torres es acero de calidad A-36 (Tensión de fluencia 250 MPa, Tensión de ro- tura $400 \mathrm{MPa}$ ); sus propiedades se consideraron linealmente elásticas y constantes en el tiempo. Las condiciones de apoyo de la torre fueron consideradas empotradas debido a que sus patas tienen apoyos independientes empotrados a la cimentación por medio de planchas de acero ancladas con pernos a la masa de hormigón y unidas con pernos a las patas de la torre.

Se realizó el análisis modal de la estructura utilizando el método de Eigenvector (Vectores Propios) que se encuentra implementado en el programa SAP200o. Las masas de las antenas fueron asignadas como masas concentradas en los puntos de ubicación de las antenas. El período del primer modo de oscilación de la torre con presencia de antenas fue de 1,25 segundos (frecuencia $0,8 \mathrm{~Hz}$ ).

\subsection{Cálculo de las cargas}

Las cargas consideradas son las cargas permanentes y la carga de viento. Las cargas permanentes son el peso de los elementos estructurales y el peso de las antenas. El peso de las antenas UHF y VHF se obtuvo del catálogo de la empresa RYMSA (23). La carga de viento sobre las antenas fue calculada a partir de la multiplicación de la presión dinámica del viento a la altura de ubicación de las antenas, el coeficiente de ráfaga, el coeficiente de forma y su área expuesta al viento. El coeficiente de forma de las antenas VHF fue obtenido mediante ensayos en túnel de viento (24), mientras que el coeficiente de forma de las antenas UHF se obtuvo de los datos que ofrece la empresa Kathrein (25).

La carga de viento fue calculada a partir de considerar como valores comunes para todas las normas: la velocidad básica del viento, el perfil de velocidades y el coeficiente de forma. Además se consideraron las características turbulentas de los vientos en ciclones a partir de establecer como parámetros comunes: la intensidad de turbulencia, la escala de la turbulencia y el espectro de turbulencia en las normas ISO, EC3 y AS/NZ. La NC fue aplicada íntegramente, ya que no es posible modificar los parámetros asociados a la turbulencia del viento.

Los perfiles de velocidades de viento que responden a la ley logarítmica y a la ley potencial son los principales modelos matemáticos para describir el comportamiento de la variación de la velocidad del viento con la altura (26). La mayoría de las normas internacionales tienen incorporados estos perfiles y así se refleja en el estudio comparativo realizado por Kwon y Kareem en el año 2013 (27). De las normas estudiadas, la norma NC emplea el perfil potencial, la internacional de viento ISO hace empleo de ambos modelos y el EC3 que referencia a la norma eurocódigo de viento (EN) (11) utiliza el perfil logarítmico al igual que la norma de AS/NZ. En zonas propensas al paso de ciclones, como es el caso de Cuba, el ajuste de los perfiles anteriores ha sido tema de estudio y cuestionamiento. Tamura y Kareem (26) exponen que aún hay insuficiente comprensión de este fenómeno producto de las pocas mediciones de campo exitosas bajo condiciones extremas. Sin embargo, se registran algunos estudios (28), (29) y (30) que a pesar de tener diferencias, coinciden que para las regiones bajas de la capa límite atmosférica (entre 100 y 300 metros en función de la referencia), tanto el perfil potencial como el logarítmico ofrecen resultados adecuados para la simulación de los regímenes de vientos producidos por los mecanismos ciclónicos. En este trabajo se decidió emplear el 

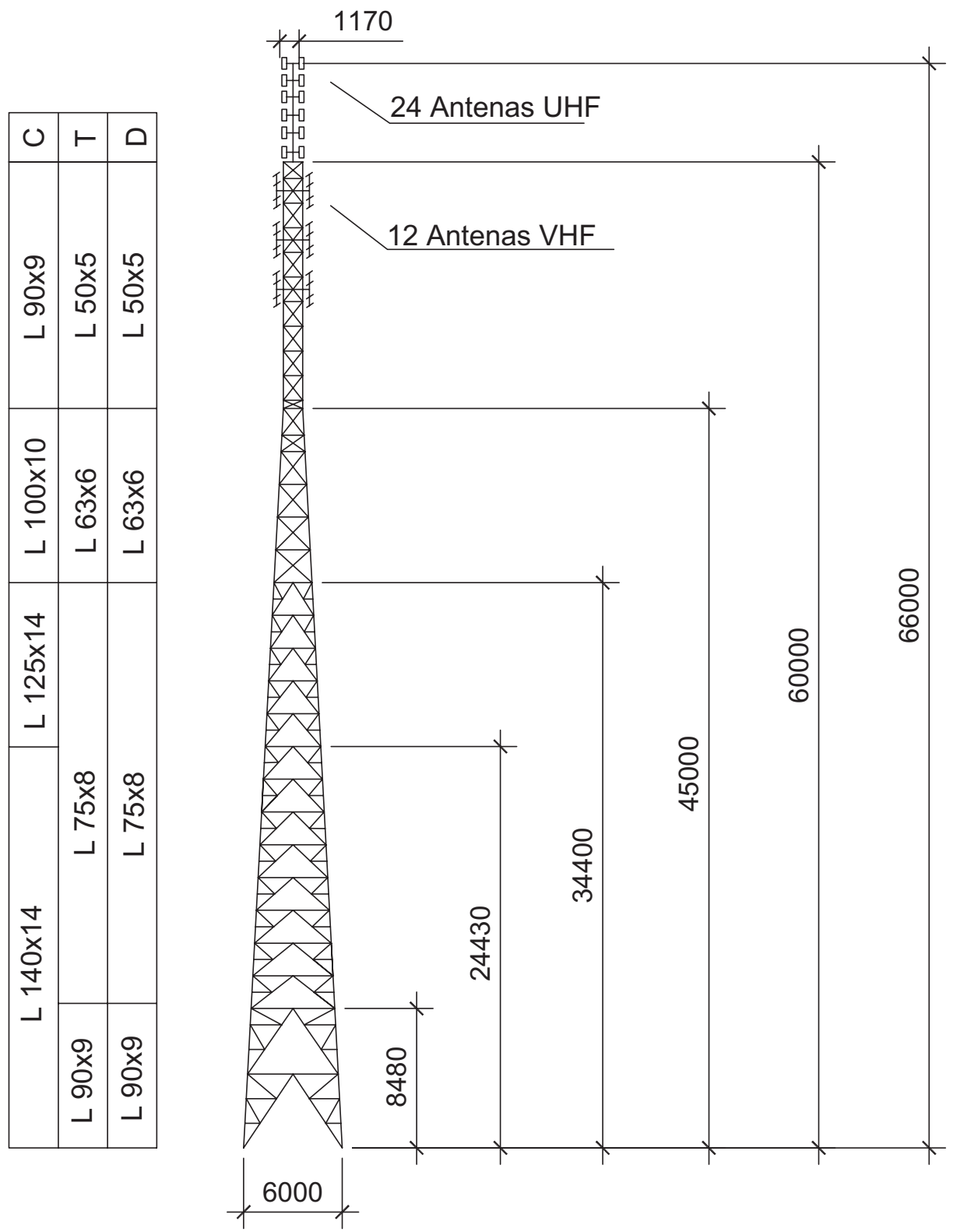

Figura 1. Características geométricas de la torre y ubicación de las antenas (dimensiones en mm).

perfil potencial independientemente de la norma foránea a aplicar.

Para la caracterización de la componente fluctuante del viento, las normas internacionales estudiadas AS/NZ, EC3, ISO definen parámetros como el espectro, la escala integral y la intensidad de turbulencia, no así la NC que no define estos parámetros. En cuanto al espectro, las normas ISO y AS/NZ emplean la formulación de von Kármán, mientras que la norma EN emplea la de Kaimal. Un grupo de estudios (26), (31), (32) y (33) avalan que la formulación de von Kármán es la representación más adecuada para la descripción de la turbulencia del viento durante el paso de tormentas ciclónicas, y por tanto fue el espectro seleccionado para este estudio. El espectro se encuentra representado en las normas a través del factor de energía espectral que se define en la sección 2.3.

Para la escala integral de la turbulencia no hay una definición concluyente sobre cuál es la formulación más apropiada, aunque existen investigaciones derivadas de mediciones en pre- sencia de ciclones en Asia (32), (34), (35). Un trabajo reciente de Li et al. (36) expone que se necesita profundizar en el estudio de este aspecto, y no propone ninguna expresión para su cálculo. Las tres normativas estudiadas emplean diferentes formulaciones para la obtención de la escala integral de la turbulencia, ver tabla 1. En la tabla 1 se muestran además los valores del parámetro de acuerdo a la torre objeto de estudio calculadas para la altura de referencia $(\boldsymbol{h}=60$ metros) y para una longitud de rugosidad $\left(\mathbf{z}_{\mathrm{o}}\right)$ igual a 0,05 , ya que la torre se encuentra ubicada en un sitio de terreno abierto.

Tabla 1. Escala longitudinal de la turbulencia.

\begin{tabular}{|c|c|c|}
\hline Norma & $\begin{array}{c}\text { Formulaciones de la escala } \\
\text { integral de la turbulencia }\left(\boldsymbol{L}_{\boldsymbol{u}}\right)\end{array}$ & $\begin{array}{c}\text { Valores } \\
\text { de } \boldsymbol{L}_{\boldsymbol{u}}\end{array}$ \\
\hline AS/NZ (11) & $85(h / 10)^{0,25}$ & $133,03 \mathrm{~m}$ \\
\hline $\mathrm{ISO}(14)$ & $100(h / 10)^{0,5}$ & $141,42 \mathrm{~m}$ \\
\hline $\mathrm{EN} \mathrm{(13)}$ & $300\left(\frac{h}{200}\right)^{0,67+0,05 \ln \left(z_{0}\right)}$ & $160,36 \mathrm{~m}$ \\
\hline
\end{tabular}


Por la variabilidad encontrada en la bibliografía estudiada se decidió emplear en este estudio la formulación para la escala de la turbulencia obtenida por Solari y Piccardo (37), que fue resultado del estudio de varias fuentes bibliográficas de diferentes regiones del planeta. La formulación propuesta por Solari y Picardo coincide con la propuesta de la norma EN.

La intensidad de turbulencia, expresada mediante la ecuación [1], también tiene diferentes enfoques en cada normativa. Las normas ISO y AS/NZ proponen tablas en función de la categoría del terreno mientras que la norma EN propone una formulación derivada del perfil logarítmico de velocidad.

$$
I_{h}=\sigma_{u} / \bar{U}
$$

Donde $\sigma_{u}$, es la desviación estándar de la velocidad y es la velocidad media.

Existen estudios realizados a través de mediciones físicas (34), (36), (38), (39) durante el paso de ciclones tropicales donde se ha podido corroborar que la mezcla turbulenta conduce a mayores valores de la intensidad de turbulencia que aquellos obtenidos a través de las normas. Li et al. (36) proponen una formulación analítica (ecuación [2]) que permite obtener el factor de intensidad de turbulencia $(\beta)$ a partir de las evidencias experimentales. El factor se relaciona con el cuadrado de la desviación estándar, varianza $(\beta)$, mediante la ecuación [3] y por ende posibilita la obtención de la intensidad de turbulencia de una forma mejor ajustada a las condiciones de ciclones.

$$
\begin{gathered}
\beta=\left(2,72-0,25 \log \left(z_{\mathrm{o}}\right)\right)^{2} \\
\sigma_{u}{ }^{2}=\beta u_{*}^{2}
\end{gathered}
$$

Donde $u_{*}$ es la velocidad de fricción que es posible obtenerla mediante la extrapolación del perfil logarítmico (ecuación [4]) a partir del conocimiento de la velocidad del viento en una altura determinada.

$$
\overline{U_{z}}=\frac{u_{*}}{k} \ln \frac{Z}{z_{\mathrm{o}}}
$$

Donde $z$ es la altura donde es calculada la velocidad $(z=10$ m), $z_{0}$ es igual a $0,05, k$ es la constante de von Kármán, aproximadamente igual a o,4 y $\bar{U}_{z}$ es la velocidad, en este caso, a 10 metros de altura desde el nivel del terreno igual a $45 \mathrm{~m} / \mathrm{s}$ (valor seleccionado de la norma NC como se muestra en la sección 2.3.1), por tanto se obtiene un valor de velocidad de fricción igual a 3,4 m/s. El valor de intensidad de turbulencia resultante para la máxima altura de la torre estudiada fue de 0,17 obtenido a partir del valor de velocidad de fricción y con el factor de intensidad de turbulencia $\beta=9,27$ calculado de acuerdo a la ecuación [2].

La torre fue dividida en diferentes tramos para la determinación de las cargas, en cuyo punto medio se consideró aplicada la carga de viento de forma concentrada sobre los cuatro nudos que se encuentran en los vértices de la sección transversal. La dirección de viento utilizada para la comparación entre los métodos propuestos por las normas seleccionadas fue o grados, o sea perpendicular a la cara de la torre.
Las combinaciones de cargas utilizadas fueron las establecidas en la norma cubana NC-450:2006 (40) (ver ecuaciones [5] y [6]). La factorización de las cargas fue aplicada a los valores de las cargas de viento obtenidos para cada una de las normas estudiadas.

$$
\begin{aligned}
& 0.9 C P+1.4 C V \\
& 1.2 C P+1.4 C V
\end{aligned}
$$

En las expresiones anteriores $\mathrm{CP}$ es la carga permanente y $\mathrm{CV}$ la carga de viento.

\subsection{Descripción de las normas estudiadas}

\subsubsection{Norma NC-285:2003}

La norma NC establece dos componentes para el cálculo de la carga de viento una estática y otra dinámica. La componente estática se obtiene a partir de la multiplicación de una presión básica por una serie de coeficientes que tienen en cuenta los parámetros meteorológicos y de forma que actúan sobre el área neta de la estructura según se muestra en la ecuación [7].

$$
Q=q_{10} * C t * C s * C h * C r * C r a * C f *\left[A_{N E T A}\right](\mathrm{kN})
$$

Donde $q_{10}$ es la presión básica obtenida a partir de una velocidad básica promediada en 10 min para un período de retorno de 50 años. La presión básica seleccionada fue $1,3 \mathrm{kN} / \mathrm{m}^{2}$ que corresponde a un valor de velocidad $\left(\bar{U}_{z}\right)$ de $45 \mathrm{~m} / \mathrm{s}$ debido a que la torre estudiada está ubicada en la región occidental de Cuba. Ct es el coeficiente de recurrencia que presenta valor igual a 1 para un período de retorno de 50 años. Cs es el coeficiente de sitio que presenta valor igual a 1 para un sitio normal. Ch es el coeficiente de altura obtenido a partir de un perfil potencial de velocidades de exponente igual a 0,16 ya que la torre se encuentra ubicado en un terreno abierto. La expresión propuesta en la norma para obtener el coeficiente de altura para este tipo de terreno es:

$$
C h=\left(\frac{z}{10}\right)^{0,32}
$$

Donde $z$ es la altura de los puntos de aplicación de la carga. El valor de 0,32 está dado porque el coeficiente afecta la presión y no la velocidad de viento.

Cr es el coeficiente de ráfaga que presenta un valor de 1,08 determinado según el tipo de terreno y la altura total de la estructura. Cra es el coeficiente de reducción de área el cuál presenta un valor de o,9 según la dimensión máxima de la estructura que corresponde a la altura de la torre. $C f$ es el coeficiente de forma, la torre seleccionada en este trabajo también fue estudiada para el análisis de sus coeficientes de forma a partir de ensayos en un túnel de viento (24), (41). Los resultados de estos estudios (24), (41) mostraron que los valores de los coeficientes de forma obtenidos mediante el ensayo en túnel de viento son similares a los propuestos en las normas cuando el ensayo se realiza bajo flujo con baja intensidad de turbulencia o flujo suave. Los valores de los coeficientes de forma obtenidos de los tramos seleccionados de la torre para el ensayo fueron generalizados a otros tramos de la torre que presentan relaciones de solidez y características geométricas similares. $A_{N E T A}$ es el área neta correspondiente al área de los 
perfiles de una cara de la torre, calculada para los tramos en que fue dividida la torre para el cálculo de la carga de viento.

La norma NC expone que la componente dinámica de la carga de viento se determina para cada forma de oscilación de la obra que presente período de oscilación mayor de un segundo. La torre objeto de estudio presenta período de oscilación de 1,25 segundos como se expuso en la sección 2.1. Los valores de la componente dinámica se determinan a partir de un esquema de fuerzas inerciales aplicadas sobre las masas concentradas en cada tramo en que fue subdividida la torre para el cálculo de la carga estática del viento. La fuerza de inercia aplicada sobre la "j" ésima masa en el "i" ésimo modo de oscilaciones propias de la obra, se determina por la ecuación [9]:

$$
Q_{J I}^{N}=M_{J} * C_{I}^{D *} C_{C E} * N_{J I}
$$

Donde $M_{J}$ es la " $j$ " ésima masa concentrada del tramo en $\mathrm{kg}$, $C_{I}^{D}$ es el coeficiente dinámico del modo "i” ésimo, $N_{J I}$ es la aceleración reducida de la "j" ésima masa, $\mathrm{m} / \mathrm{s}^{2}$ y $C_{C E}$ es el coeficiente que tiene en cuenta la correlación espacial de las pulsaciones según la altura y fachada de la torre. Las solicitaciones totales de la estructura sometidas a la acción de la carga de viento se determinan por la ecuación [10].

$$
X_{V}^{*}=\gamma_{s} X^{E}+\sqrt{\sum_{t=1}^{s}\left(X_{i}^{D}\right)^{2}}
$$

Donde el término $X_{V}^{*}$ es el valor de la solicitación de cálculo total debido al efecto de las cargas de viento; es el valor de la solicitación debido a la componente estática de la carga de viento; $X_{i}^{D}$ valor de la solicitación debido a la componente dinámica de la carga de viento en cada modo de oscilación considerado, $s$ es el número de modos de oscilaciones considerado. $\gamma_{s}$ es el coeficiente de mayoración de la carga tomado igual a 1,4 como se mostró anteriormente en las ecuaciones [5] y [6] de combinaciones de carga.

\subsubsection{Normas ISO 4354-2009, AS/NZS1170.2-2011 y EC 3: 2007}

La expresión general para el cálculo de la fuerza de viento sobre la torre según las normas ISO, AS/NZ y EC3 se muestra en la ecuación [11].

$$
F=q_{z} C_{f} G A_{r e f}
$$

Donde $q_{z}$ es la presión dinámica del viento a la altura $z, C_{f}$ es el coeficiente de forma, $G$ es el factor de efecto de ráfaga y $A_{r e f}$ es el área de referencia. El área de referencia es el área neta correspondiente al área de los perfiles de una cara de la torre. Los valores de coeficiente de forma y área neta son los mismos que los utilizados en el cálculo de la carga de viento según la NC.

La presión dinámica $\left(q_{z}\right)$ puede ser generalizada a partir de la expresión que proponen Kwon y Kareem (30):

$$
q_{z}=\frac{1}{2} \rho\left(U \cdot C_{\text {exposición }}\right)^{2} \cdot C_{\text {topografia }} \cdot C_{\text {dirección }} \cdot C_{\text {importancia }} \cdot C_{\text {otros }}
$$

Donde $\rho$ es la densidad del aire $\left(\rho=1,225 \mathrm{~kg} / \mathrm{m}^{3}\right)$, es el valor de la velocidad básica $(U=45 \mathrm{~m} / \mathrm{s}$ promedio en 10 minutos, igual al utilizado en la NC), $C_{\text {exposición }}$ es el coeficiente asociado al perfil de velocidad o coeficiente de exposición $\left(C_{\text {exposición }}=\right.$ $\left.(z=10)^{0,16}\right), C_{\text {topografia }}$ es el coeficiente topográfico, $C_{\text {dirección }}$ es el coeficiente de la dirección del viento, $C_{\text {importancia }}$ es el coeficiente de importancia de la edificación, $C_{\text {otros }}$ es el coeficiente que tiene en cuenta otras características tales como zonas de ciclón, efectos de protección, variaciones en el período de retorno. En este trabajo los coeficientes de topografía, importancia, direccionalidad son iguales a 1 . El coeficiente $C_{\text {otros }}$ toma valor igual a 1, ya que no se consideraron las variaciones en el período de retorno, la consideración de ubicación de la torre en zona de ciclón se tiene en cuenta en el valor de la velocidad básica ya que este fue obtenido a partir una serie de datos de velocidades que incluyen las velocidades de ciclones en Cuba.

El factor de efecto de ráfaga $(G)$ puede ser generalizado como muestra la ecuación [13]:

$$
G=\frac{G L F}{G_{q}}
$$

Donde $G L F$ es el factor respuesta de ráfaga y $G_{q}$ es el factor de ráfaga para la presión del viento. $G_{q}$ se utiliza para equilibrar las diferencias entre los diferentes intervalos de promediación: de la velocidad básica del viento y de la respuesta de la estructura. Las tres normas ISO, AS/NZ y EC3 presentan intervalos de la respuesta al viento de 10 minutos mientras que el intervalo de promediación de la velocidad básica es de 10 minutos para las normas ISO y EC3 y 3 segundos para la AS/NZ. En este trabajo se utilizó un solo valor de velocidad básica promediado en $10 \mathrm{minu-}$ tos en correspondencia con los valores de velocidad básica utilizados en la NC. Como los intervalos de promediación de la velocidad y de la respuesta son iguales, el valor $G_{q}$ de es igual a la unidad. GLF fue definido por Davenport (1) como:

$$
G=1+r \sqrt{g_{B}^{2} B+g_{R}^{2} R}
$$

Donde $r$ se define como dos veces el valor de la intensidad de turbulencia $\left(2 I_{h}\right), B$ y $R$ son los factores de respuesta no resonantes y resonantes respectivamente, $g_{B}$ y $g_{R}$ son los factores picos de las respuestas no resonante y resonante.

El factor de efecto de ráfaga $G$ es denominado como $C_{d y n}$ en las normas ISO (Método basado en la velocidad media) y AS/NZ, se define como se muestra en las ecuaciones [15] $\mathrm{y}$ [16] respectivamente.

$$
\begin{gathered}
C_{d y n, m}=1+2 I_{v h} \sqrt{g_{D B}^{2} B_{D}+g_{D R}^{2} R_{D}^{2}} \\
C_{d y n}=\frac{1+2 I_{h} \sqrt{g_{v}^{2} B_{s}+\frac{H_{s} g_{R}^{2} S E_{t}}{\zeta}}}{1+2 g_{v} I_{h}}
\end{gathered}
$$

El denominador de la ecuación [16] representa el valor de $G_{q}$ ya que el intervalo de promediacion de la velocidad básica es de 3 segundos en la AS/NZ. En este trabajo $G_{q}$ toma valor igual a 1 según lo expuesto anteriormente. $H_{s}$ es el factor de altura para la respuesta resonante que se define como: 


$$
H_{s}=1+\left(\frac{s}{h}\right)^{2}
$$

Donde $s$ es la altura medida desde la base hasta el punto de aplicación de la carga y $h$ es la altura total de la torre.

La norma EC3 propone para obtener la carga de viento de ráfaga equivalente, la multiplicación de la carga media del viento por el siguiente término, que es equivalente al factor de efecto de ráfaga $G$ :

$$
1+\left(1+0.2\left(z_{m} / h\right)^{2}\right)\left(\left[1+7 I_{v}\left(z_{e}\right)\right] c_{s} c_{d}-1\right)
$$

Donde $z_{m}$ es la altura medida desde la base hasta el punto de aplicación de la carga, $h$ es la altura total de la torre, $c_{s} c_{d}$ es el factor estructural que se define según la norma EC como:

$$
c_{s} c_{d}=\frac{1+2 \cdot k_{p} \cdot I_{v}\left(z_{e}\right) \cdot \sqrt{B^{2}+R^{2}}}{1+7 I_{v}\left(z_{e}\right)}
$$

En la norma EC3 la fuerza media está planteada en función de la presión dinámica de ráfaga, por esta razón es dividida luego entre $G_{q}$, que está representado por el denominador de la ecuación [19]. Como se mencionó anteriormente, en este trabajo se utilizan los valores de presiones medias basados en una velocidad básica promediada en 10 minutos, por lo que $G_{q}$ toma valor de 1 .

En las ecuaciones [15], [16] y [18] los términos $I_{v h}, I_{h}$ e $I_{v}$ son la intensidad de turbulencia. Los factores de la respuesta no resonante $\left(B_{D}, B_{s}\right.$ y $\left.B\right)$ están definidos según cada norma en la tabla 2. La respuesta resonante se expresa generalmente, como una función del factor de reducción de tamaño $(S)$, el factor de energía espectral $(E)$ y la razón de amortiguamien-

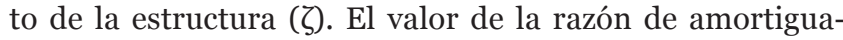
miento utilizado es de 0,05. En las normas ISO y EN estos parámetros se tienen en cuenta en el factor de respuesta resonante $\left(R_{D}\right.$ y $\left.R\right)$, sin embargo la norma AS/NZ define estos parámetros directamente en la ecuación del factor de ráfaga. Las expresiones para la obtención de los factores de la respuesta resonante según cada norma se muestran en la tabla 3.
En la tabla $3 E_{D}, E$ y $S_{L}$ son los factores de energía espectral asumidos mediante la formulación de von Kármán según lo planteado en la sección 2.2, ver ecuación [20].

$$
E=\frac{4 f L_{u} / \bar{U}_{z}}{\left[1+70.8\left(f L_{u} / \bar{U}_{z}\right)^{2}\right]^{5 / 6}}
$$

De la ecuación [20], es la frecuencia de oscilación del modo fundamental de vibración de la estructura, $\bar{U}_{z}$ es la velocidad media del viento a la altura de referencia y $L_{u}$ es la escala longitudinal de la turbulencia calculada para la altura de referencia según se definió en la sección 2.2.

Los factores picos de la respuesta resonante y no resonante de la ecuación [14] tienen valores o formulaciones diferentes en cada normativa. En la norma ISO el factor $g_{R}$ se denomina $g_{D R}$ y se obtiene por la ecuación [21], en la norma AS/NZ se plantea la ecuación [22] y en la norma EC3 asume la terminología de $k_{p}$ y se calcula mediante la ecuación [23]. Para los factores $g_{B}$ en la norma ISO se asume valor de 3,4, en la norma $\mathrm{AS} / \mathrm{NZ}$ de 3,7 y en la norma $\mathrm{EC}_{3}$ toma el mismo valor de $k_{p}$.

$$
\begin{gathered}
g_{D R}=\sqrt{2 \ln (v T)}+0.5772 / \sqrt{2 \ln (v T)} \\
g_{R}=\sqrt{\left[2 * \ln \left(T n_{a}\right)\right]} \\
k_{p}=\sqrt{2 \ln (v T)}+0.6 / \sqrt{2 \ln (v T)}
\end{gathered}
$$

Para las tres ecuaciones anteriores, $T$ representa el intervalo de promediación de la respuesta, igual a 600 segundos. De la ecuación [21], $v$, y de la [22], $n_{a}$, son la frecuencia natural del primer modo de oscilación de la estructura (Hz). En la ecuación [23] se obtiene a través de la ecuación [24], donde es la frecuencia del primer modo de oscilación.

\begin{tabular}{|c|c|c|c|}
\hline Norma & & & $B$ \\
\hline ISO & $B_{D}$ & $\frac{1+0,2 \beta}{1+\frac{0,63\left(\frac{\sqrt{b h}}{L_{v h}}\right)^{0.56}}{\left(\frac{h}{b}\right)^{\gamma}}}$ & $\begin{array}{l}\beta: \text { exponente de la ley potencial del perfil de velocidades de viento. } \\
b: \text { ancho de la estructura (fue seleccionado el ancho promedio) } \\
h: \text { altura de referencia } \\
L_{v h}: \text { escala integral de la turbulencia a la altura de referencia } \\
\gamma 0,07 \text { para } h / b \geq 1\end{array}$ \\
\hline $\mathrm{AS} / \mathrm{NZ}$ & $B_{s}$ & $\frac{1}{1+\frac{0,26(h-s)^{2}+0,46 b_{s h}^{2}}{L_{h}}}$ & $\begin{array}{l}b_{s h}: \text { ancho promedio del tramo analizado } \\
h: \text { altura total de la estructura } \\
s: \text { altura medida desde la base hasta el punto de aplicación de la carga } \\
L_{h}: \text { escala integral de la turbulencia }\end{array}$ \\
\hline $\mathrm{EN}$ & $B$ & $\sqrt{\frac{1}{1+0,9\left(\frac{b+h}{L_{(z)}}\right)^{0,63}}}$ & $\begin{array}{l}b: \text { ancho de la estructura (fue seleccionado el ancho promedio) } \\
h: \text { altura de la estructura } \\
L_{(z)}: \text { escala integral de la turbulencia }\end{array}$ \\
\hline
\end{tabular}

$$
v=n \sqrt{\frac{R^{2}}{B^{2}+R^{2}}}
$$

La norma australiana específica de torres AS3995-1994 (17) y el EC3 definen patrones de carga para obtener las fuerzas en los elementos diagonales ubicados en los primeros tramos

Tabla 2. Factores de la respuesta no resonante. 
Tabla 3. Factores de la respuesta resonante.

\begin{tabular}{|c|c|c|c|}
\hline Norma & & & $\boldsymbol{R}$ \\
\hline ISO & $R_{D}$ & $(1+0,6 \beta) \frac{3}{2+k} K \sqrt{\frac{\pi}{4 \zeta_{D}}} E_{D} S(0,57+0,35 \beta+r)$ & $\begin{array}{l}\beta: \text { exponente de la ley potencial del perfil de velocidad } \\
K \text { : factor de corrección del modo de oscilación, calculado como } \\
k: \text { exponente de la forma del modo de oscilación } \\
\zeta_{D}: \text { razón de amortiguamiento } \\
E_{D}: \text { factor de energía espectral } \\
S=\frac{0,9}{1+6\left(\frac{f_{D} h}{V_{\text {sitio, }}}\right)^{2}+\left(1+3\left(\frac{f_{D} b}{V_{\text {sitio, },}}\right)\right)} \\
r=\frac{2 \sqrt{0,053-0,042 \beta}}{1+20\left(\frac{f_{D} b}{V_{\text {sitio,m }}}\right)} \\
V_{\text {sitio, }}: \text { velocidad media en el sitio }\end{array}$ \\
\hline $\mathrm{AS} / \mathrm{NZ}$ & $\frac{S E}{\zeta}$ & $S=\frac{1}{\left[1+\left[\frac{3,5 n_{a} h}{\bar{V}_{h}}\right]\left[1+\frac{4 n_{a} b_{o h}}{\bar{V}_{h}}\right]\right]}$ & $\begin{array}{l}\bar{V}_{h}: \text { velocidad media en el sitio } \\
b_{o}: \text { ancho promedio de la estructura } \\
h: \text { altura total de la estructura } \\
s: \text { altura medida desde la base hasta el punto de aplicación de la } \\
\text { carga } \\
E: \text { factor de energía espectral }\end{array}$ \\
\hline EN & $R$ & $\sqrt{\left(\frac{\pi^{2}}{2 \delta} S_{L}(s, n) R_{h}\left(\eta_{h}\right) R_{b}\left(\eta_{b}\right)\right)}$ & $\begin{array}{l}R_{h}=\frac{1}{\eta_{h}}-\frac{1}{2 \eta_{h}^{2}}\left(1-e^{-2 \eta_{h}}\right) \\
R_{b}=\frac{1}{\eta_{b}}-\frac{1}{2 \eta_{b}^{2}}\left(1-e^{-2 \eta_{b}}\right) \\
\eta_{h}=\frac{4 \cdot 6 h}{L(z)} f_{L}(z, n) \\
\eta_{b}=\frac{4 \cdot 6 b}{L(z)} f_{L}(z, n) \\
f_{L}=\frac{n L(z)}{V_{m}(z)} \\
V_{m}(z): \text { velocidad media del sitio } \\
S_{L}: \text { factor de energía espectral } \\
L_{(z)}: \text { escala integral de la turbulencia }\end{array}$ \\
\hline
\end{tabular}

de la altura de la torre ya que estos elementos son sensibles a las fluctuaciones de la carga de viento. La ubicación de los patrones depende del vértice donde se intercepta la proyección de las columnas de la torre, que es 53,5 metros en la torre objeto de estudio. La norma $\mathrm{EC}_{3}$ define que si el punto de intercepción se encuentra dentro de la altura de la torre se deben aplicar dos patrones de carga: a) aplicar la carga media del viento por debajo del punto de intercepción y aplicar la carga de viento de ráfaga equivalente por encima del punto de intercepción, b) aplicar la carga media del viento por encima del punto de intercepción y aplicar la carga de viento de ráfaga equivalente por debajo del punto de intercepción. La norma AS3995-1994 (17) expone que existen dos fuerzas de viento resultantes: una fuerza resultante ubicada por debajo del punto de intercepción $\left(W_{\mathrm{u}}\right)$ y otra ubicada por encima $\left(W_{\mathrm{u} 2}\right)$. Los patrones de carga que define la norma $\mathrm{AS}_{3995}$ 1994 son: a) $W_{\mathrm{u} 1}+W_{\mathrm{u} 2}$, b) $0,5 W_{\mathrm{u} 1}+W_{\mathrm{u} 2}$, c) $W_{\mathrm{u} 1}+0,5 W_{\mathrm{u} 2}$. En este trabajo fueron aplicados los patrones de cargas para la obtención de las fuerzas en los elementos de la torre según lo establecido en las normas EC3 y AS3995-1994 (17).

\section{RESULTADOS Y DISCUSIÓN}

La tabla 4 muestra los valores de los parámetros geométricos de la torre: altura de referencia $\left(h_{\text {ref }}\right)$ y ancho promedio $(b)$. Además la tabla 4 presenta los factores picos de las respues- tas no resonante $\left(g_{B}\right)$ y resonante $\left(g_{R}\right)$, así como el factor de efecto de ráfaga $(G)$ calculados según las ecuaciones propuestas en las normas ISO, AS/NZ y EC3. Como se observa en la tabla 4, la norma AS/NZ presentó mayores valores de $G$ del orden de $4 \%$ y $9 \%$ con respecto a las normas EC3 e ISO respectivamente.

La comparación entre las normas ISO, AS/NZ y EC3 con la norma NC estuvo basada en las diferencias que existen entre los valores máximos de las fuerzas axiales en los elementos de la torre (columnas, tranques y diagonales) y en los desplazamientos máximos. Los valores máximos de las fuerzas en los elementos diagonales fueron obtenidos a partir de la aplicación de los patrones de carga definidos en las normas EC3 y AS3995-1994 (17). Los elementos de la torre fueron subdivididos en varios tramos según variaban las dimensiones de las secciones de los perfiles con la altura, tomando como tramo 1 el tramo más cercano a la base de la torre.

La tabla 5 muestra los máximos valores de las fuerzas axiales tanto a tracción como a compresión registrados en los tramos de las columnas, diagonales y tranques, en la tabla la letra C indica compresión y la T indica tracción. En esta tabla se evidencia que los valores de fuerzas axiales obtenidos de la aplicación de las normas ISO, EC3 y AS/NZ son mayores que los obtenidos de la aplicación de la norma NC. De la tabla 5 pue- 
Tabla 4. Parámetros geométricos, factores picos y factores de efecto de ráfaga

\begin{tabular}{|c|c|c|c|}
\hline Parámetros & ISO & AS/NZ & EC 3 \\
\hline$h_{r e f}(m)$ & 60 & 60 & 60 \\
\hline$b(m)$ & 3,85 & 3,85 & 3,85 \\
\hline$g_{B}$ & 3,4 & 3,7 & 3,63 \\
\hline$g_{R}$ & 3,68 & 3,51 & 3,63 \\
\hline$G$ & 2,24 & 2,45 & 2,35 \\
\hline
\end{tabular}

Tabla 5. Comparación entre las normas para las fuerzas axiales en columnas, diagonales y tranques.

\begin{tabular}{|c|c|c|c|c|c|c|c|c|c|c|c|c|c|}
\hline \multirow{2}{*}{ Tramos } & \multirow{2}{*}{$\begin{array}{l}\text { Tipo de } \\
\text { F. axial }\end{array}$} & \multicolumn{4}{|c|}{ F. Columnas (kN) } & \multicolumn{4}{|c|}{ F. Diagonales (kN) } & \multicolumn{4}{|c|}{ F. Tranques $(\mathrm{kN})$} \\
\hline & & NC & ISO & AS/NZ & EC3 & NC & ISO & AS/NZ & EC & NC & ISO & AS/NZ & EC \\
\hline \multirow{2}{*}{1} & $\mathrm{C}$ & 1044 & 1533 & 1746 & 1603 & 80 & 145 & 167 & 147 & 36 & 72 & 83 & 72 \\
\hline & $\mathrm{T}$ & 943 & 1433 & 1645 & 1503 & 76 & 141 & 163 & 143 & 36 & 71 & 83 & 72 \\
\hline \multirow{2}{*}{2} & $\mathrm{C}$ & 767 & 1033 & 1164 & 1093 & 49 & 88 & 103 & 90 & 50 & 65 & 72 & 69 \\
\hline & $\mathrm{T}$ & 714 & 980 & 1111 & 1040 & 46 & 86 & 100 & 87 & 52 & 67 & 72 & 71 \\
\hline \multirow{2}{*}{3} & $\mathrm{C}$ & 631 & 804 & 895 & 853 & 109 & 134 & 146 & 141 & 107 & 135 & 149 & 143 \\
\hline & $\mathrm{T}$ & 600 & 774 & 864 & 823 & 105 & 130 & 142 & 137 & 111 & 139 & 154 & 145 \\
\hline \multirow[b]{2}{*}{4} & $\mathrm{C}$ & 624 & 768 & 837 & 810 & 62 & 86 & 88 & 87 & 57 & 71 & 77 & 75 \\
\hline & $\mathrm{T}$ & 532 & 660 & 719 & 696 & 62 & 86 & 87 & 87 & 59 & 73 & 80 & 77 \\
\hline
\end{tabular}

de observarse que las mayores diferencias entre los valores de las fuerzas obtenidas por las normas ISO, EC3 y AS/NZ respecto a la norma NC se encuentran en el primero y segundo tramo de la torre. En estos dos tramos, los incrementos relativos de las normas ISO, AS/NZ y EC3 respecto a la norma NC oscilan entre $34-74 \%$ para las fuerzas en las columnas y en las diagonales las fuerzas axiales pueden llegar a ser el doble que aquellas obtenidas por la norma NC. En los tramos tres y cuatro los incrementos son del orden de $23-44 \%$ para las fuerzas en columnas y para las diagonales entre $22-42 \%$. En las fuerzas axiales de los tranques del primer tramo se alcan- zan valores por las normas ISO, EC3 y AS/NZ que superan en el doble a los obtenidos por la norma NC. En los tramos restantes los incrementos relativos de las normas ISO, AS/NZ y EC3 respecto a la norma NC son del orden de 23-44\%. Estos incrementos registrados pueden estar asociados no solo al método de análisis de la carga de viento, sino también a los parámetros relacionados con la componente fluctuante de la velocidad del viento; que pudieron ser ajustados de acuerdo a las características del viento en zonas ciclónicas en las normas ISO, EC3 y AS/NZ a la luz de publicaciones recientes, no así en la norma NC. En el caso de la norma NC llama la aten-

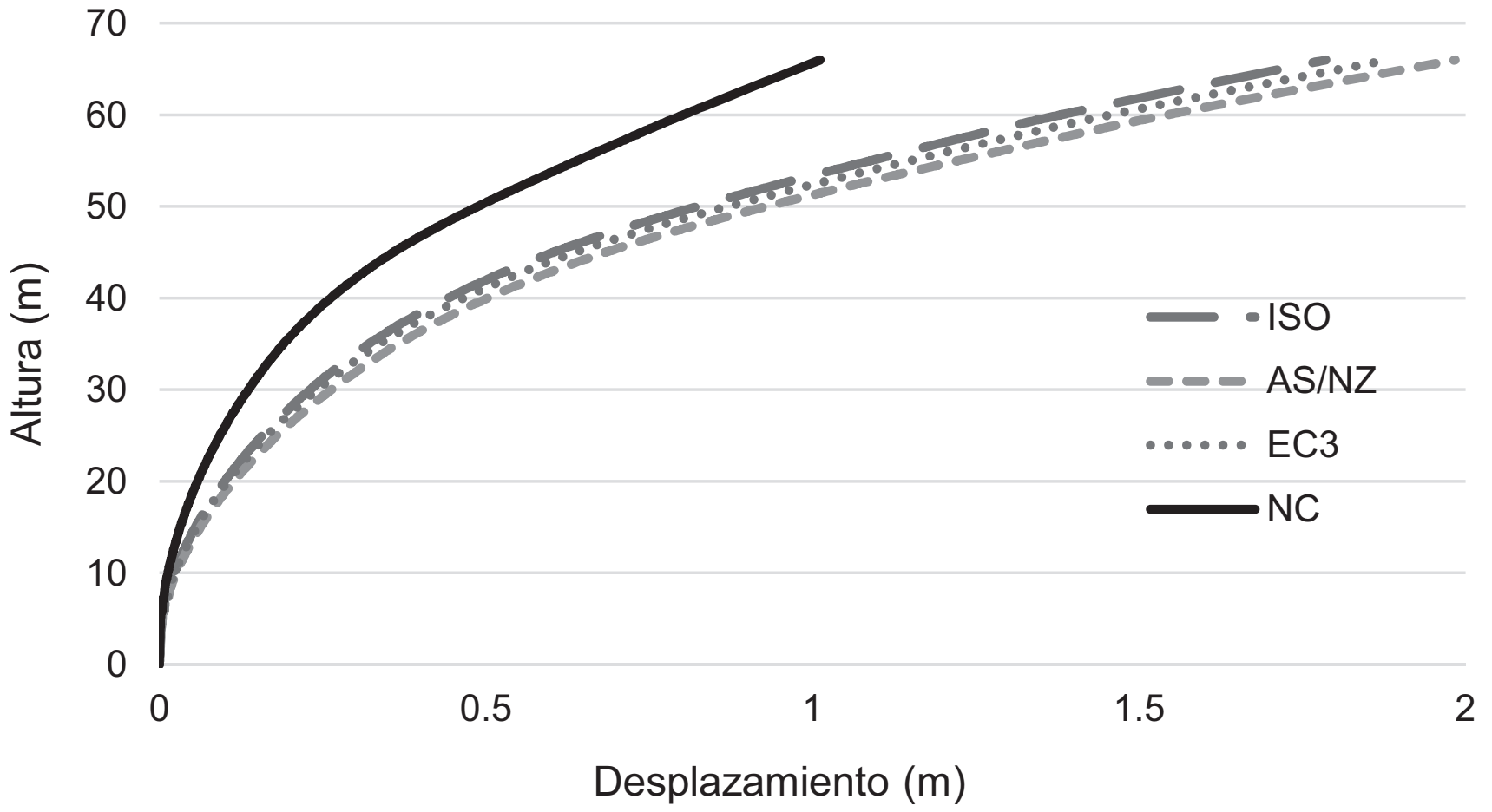

Figura 2. Comparación de los desplazamientos de la torre obtenidos para cada norma. 
ción la pequeña magnitud del coeficiente de ráfaga que afecta a la presión $(1,08)$, si se recalcula este valor teniendo en cuenta los valores de intensidad de turbulencia $(0,17)$ y factor pico de velocidad $(3,4)$, el coeficiente sería de 2,15. De forma general la norma AS/NZ es la que aporta los mayores valores de fuerzas axiales en todos los elementos, aunque sus valores son próximos a los obtenidos por las normas ISO y EC3, incluso cuando la norma ISO es una norma específica para edificaciones y no presenta las particularidades del análisis de las torres autosoportadas, por ejemplo las definiciones de los patrones de cargas. Los máximos incrementos relativos de las normas EC3 y AS/NZ con relación a la norma ISO son del $7 \%$ y $17 \%$ respectivamente.

La Figura 2 muestra los valores de los desplazamientos en función de la altura para cada una de las normas, donde se observa que el comportamiento es el mismo independientemente de la norma empleada. Se evidencia una marcada diferencia entre los valores de la norma $\mathrm{NC}$ respecto a las normas ISO, AS/NZ y EC3, que se hace más considerable con la altura, pues hasta los 15 metros de altura aproximadamente, los cuatro gráficos se solapan. Las normas ISO, EC3 y AS/NZ presentan resultados similares de desplazamientos en toda la altura de la estructura.

\section{CONCLUSIONES}

1. El método más aplicado internacionalmente, para el análisis de la respuesta longitudinal de las torres autosoportadas bajo carga de viento es el método "Factor de Efecto de Ráfaga”, implementado en las normas ISO, AS/NZ, EC3. Sin embargo no es uno de los métodos reconocidos en la norma NC por lo que se recomienda su inclusión.

2. La comparación entre las normas ISO, AS/NZ, EC3 y NC arrojó como resultado que las fuerzas axiales calculadas por la norma NC son menores que las obtenidas por las restantes normas. En algunos elementos tranques y diagonales las fuerzas axiales obtenidas por las normas ISO, AS/NZ y EC3 son dos veces mayores que los valores obtenidos por la norma $\mathrm{NC}$.

3. La norma ISO, a pesar de no presentar las particularidades del análisis de las torres autosoportadas, ofrece resultados próximos a las normas EC3 y AS/NZ. Los máximos incrementos relativos de la $\mathrm{EC} 3$ y el AS/NZ con relación a la ISO son del $7 \%$ y $17 \%$ respectivamente.

4. Se recomienda profundizar en el estudio de los valores de los coeficientes de ráfagas de la norma $\mathrm{NC}$ a partir de las consideraciones asociadas a la turbulencia del viento en regiones ciclónicas.

\section{REFERENCIAS}

(1) Davenport, A.G. (1967). Gust loading factors. Journal of Structural Division. ASCE, 93(ST-3, Paper 5255): 11-34, ISSN 0044-8001.

(2) Loredo-Souza, A.M., Davenport, A.G. (2003). The influence of the design methodology in the response of transmission towers to wind loading. Journal of Wind Engineering and Industrial Aerodynamics, 91:995-1005, doi: http://dx.doi. org/10.1016/So167-6105(03)00048-5.

(3) Zhou, Y., Kareem, A. (2001). Gust Loading Factor: New Model. Journal of Structural Engineering, 127(2): 168-175, doi: http://dx.doi.org/10.1061/(ASCE)0733-9445(2001)127:2(168).

(4) Davenport, A.G. (1964). The buffeting of large superficial structures by atmospheric turbulence. Annals of the New York Academy of Sciences, 116(1): 135-160, doi: 10.1111/j.1749-6632.1964.tb33943.x.

(5) Madugula, M.K.S. (2002). Dynamic Response of Lattice Tower and Guyed Mast, p. 110-166, Virginia, Ed. ASCE.

(6) Simiu, E. (1976). Equivalent static wind loads for tall buildings design. Journal of Structural Division, ASCE, 102: 719737.

(7) Solari, G. (1990). A Generalized Definition of Gust Factor. Journal of Wind Engineering and Industrial Aerodynamics, 36(1): 539-548, doi:dx.doi.org/10.1016/0167-6105(90)90336-B.

(8) AIJ (2004). RLB Recommendations for loads on buildings. Structural Standards Committee. Tokyo, Japan (AIJ).

(9) AS/NZS1170.2-2011 (2011). Structural Design actions, Part 2: Wind Actions. Australian/New Zeland Standart (AS/ $\mathrm{NZS})$.

(10) ASCE7-10 (2010). Minimum Design Loads for Buildings and Other Structures. American Society of Civil Engineers (ASCE).

(11) EN1991-1-4 (2004). Eurocode 1: Actions on structures - General actions - Part 1-4: Wind actions (EN).

(12) ISO4354 (2009). Wind action on structures. International Organization for Standardization (ISO).

(13) National Research Council of Canada (2010). National Building Code of Canada (NBCC), Volume 2.

(14) Holmes, J.D. (1994). Along-wind response of lattice towers: Part I-Derivation of expressions for gust response factors. Engineering Structures, 16(4): 287-292, doi:dx.doi.org/10.1016/0141-0296(94)90069-8.

(15) Holmes, J.D. (1996). Along-wind response of lattice towers II. Aerodynamic damping and deflections. Engineering Structures, 18(7): 483-488, doi:dx.doi.org/10.1016/0141-0296(95)00131-X.

(16) Smith, B.W. (2007). Communication structures, p. 148, London, Ed. A. Thomas Telford.

(17) AS3995-1994 (1994). Australian Standard: Design of steel lattice towers and masts (AS).

(18) EC3 (2007). Eurocode 3: "Design of steel structures - Part 3-1: Towers, masts and chimeneys-Towers and masts" (EC).

(19) CSA S37-01 (2001). Canadian Standards Association, Antennas, towers, and antenna supporting structures, Rexdale, Canada (CSA).

(20) TIA/EIA-222-G (2005). Structural standards for Steel Antenna Towers and Antenna Supporting Structures. Telecommunications Industry Association (TIA).

(21) NC-285 (2003). Carga de viento. Método de cálculo. Oficina Nacional de Normalización (NC).

(22) SNiP2.01.07-85 (2001). Loads and effects. State Building Committee of USSR (Gosstroi of USSR).

(23) RYMSA (2012). Broadcast products, www.rymsa.com. 
(24) Martín Rodríguez, P. (2014). Estudio analítico-experimental de torre autosoportada con presencia de antenas bajo la acción del viento (Tesis de Doctorado). La Habana: CUJAE.

(25) KATHREIN (2010). Base Station Antennas, Filters, Combiners and Amplifiers for Mobile Communications, K.-W. KG, Rosenheim, Germany.

(26) Tamura, Y., Kareem, A. (2013). Advanced Structural Wind Engineering, p.6-11, 16-18, 263-268, Japan: Springer.

(27) Kwon, D.K. and Kareem, A. (2013). Comparative study of major international wind codes and standards for wind effects on tall buildings. Engineering Structures, 51(0): 23-35, doi: 10.1016/j.engstruct.2013.01.008

(28) Vickery, P. J., Wadhera, D., Powell, M. D., \& Chen, Y. (2009). A Hurricane Boundary Layer and Wind Field Model for Use in Engineering Applications. Journal of Applied Meteorology and Climatology, 48(2), 381-405, http://dx.doi. org/10.1175/2008jamc1841.1

(29) Giammanco, I. M., Schroeder, J. L., \& Powell, M. D. (2012). Observed characteristics of tropical cyclone vertical wind profiles. Wind and Structures, 15(1), 65-86, http://dx.doi.org/10.12989/was.2012.15.1.065

(30) Tse, K. T., Li, S. W., Chan, P. W., Mok, H. Y., \& Weerasuriya, A. U. (2013). Wind profile observations in tropical cyclone events using wind-profilers and doppler SODARs. Journal of Wind Engineering and Industrial Aerodynamics, 115(o): 93-103, http://dx.doi.org/10.1016/j.jweia.2013.01.003

(31) Tamura, Y., Cao, S., \& Giang, L. T. (2012). Wind characteristics of strong tropical cyclones. Paper presented at the VI National Conference on Wind Engineering, Roorkee at New Delhi, India

(32) Li, Q.S., Zhi, L., Hu, F. (2010). Boundary layer wind structure from observations on a 325 m tower. Journal of Wind Engineering and Industrial Aerodynamics, 98(12): p. 818-832, http://dx.doi.org/10.1016/j.jweia.2010.08.001

(33) Fu, J.Y., Wu, J.R., Xu, A., Li, Q.S., Xiao, Y.Q. (2012). Full-scale measurements of wind effects on Guangzhou West Tower. Engineering Structures, 35: 120-139, http://dx.doi.org/10.1016/j.engstruct.2011.10.022

(34) Wang, B., Hu, F., \& Cheng, X. (2011). Wind gust and turbulence statistics of typhoons in South China. Acta Meteorologica Sinica, 25(1): 113-127, http://dx.doi.org/10.1007/s13351-011-0009-8

(35) Shiau, B.S. (2000). Velocity spectra and turbulence statistics at the northeastern coast of Taiwan under high-wind conditions. Journal of Wind Engineering and Industrial Aerodynamics, 88(2-3), 139-151, http://dx.doi.org/10.1016/So1676105(00)00045-3

(36) Li, L., Kareem, A., Xiao, Y., Song, L., \& Zhou, C. (2015). A comparative study of field measurements of the turbulence characteristics of typhoon and hurricane winds. Journal of Wind Engineering and Industrial Aerodynamics, 14O: 4966, http://dx.doi.org/10.1016/j.jweia.2014.12.008

(37) Solari, G., \& Piccardo, G. (2001). Probabilistic 3-D turbulence modeling for gust buffeting of structures. Probabilistic Engineering Mechanics, 16(1), 73-86, http://dx.doi.org/10.1016/So266-8920(00)0o010-2

(38) Li, L., Xiao, Y., Kareem, A., Song, L., \& Qin, P. (2012). Modeling typhoon wind power spectra near sea surface based on measurements in the South China sea. Journal of Wind Engineering and Industrial Aerodynamics, 104-106: 565-576, http://dx.doi.org/10.1016/j.jweia.2012.04.005

(39) Masters, F. J., Tieleman, H. W., \& Balderrama, J. A. (2010). Surface wind measurements in three Gulf Coast hurricanes of 2005. Journal of Wind Engineering and Industrial Aerodynamics, 98(10-11): 533-547, http://dx.doi.org/10.1016/j. jweia.2010.04.003

(40) NC450 (2006). Edificaciones-Factores de Carga o Ponderación-Combinaciones. Oficina Nacional de Normalización (NC).

(41) Martín, P., et al. (2016). Experimental study of the effects of dish antennas on the wind loading of telecommunication towers. Journal of Wind Engineering and Industrial Aerodynamics, 149: p. 40-47, http://dx.doi.org/10.1016/j. jweia.2015.11.010 